\title{
Trichoderma spp. NA EMERGÊNCIA E CRESCIMENTO DE MUDAS DE CAMBARÁ (Gochnatia polymorpha (Less.) Cabrera) ${ }^{1}$
}

\author{
Daniele Franco Martins Machado ${ }^{2}$, Antonio Padilha Tavares ${ }^{3}$, Sidinei José Lopes ${ }^{4}$ e Antonio Carlos \\ Ferreira da Silva ${ }^{5}$
}

\begin{abstract}
RESUMO - Neste trabalho, o objetivo foi avaliar o efeito de isolados de Trichoderma spp. na emergência de plântulas e no crescimento de mudas de cambará (Gochnatia polymorpha). Utilizou-se, em casa de vegetação, substrato esterilizado e não esterilizado, sendo avaliados os efeitos de quatro isolados de trichoderma: TSM1 e TSM2 de Trichoderma viride, 2B2 e 2B22 de Trichoderma harzianum mais um mix preparado com a mistura desses quatro isolados, além de dois produtos comerciais à base de trichoderma. A análise dos dados permitiu concluir que os isolados de trichoderma testados não interferem na emergência das plântulas, mas os isolados 2B2 e 2B22 de T. harzianum apresentam potenciais como promotores de crescimento de mudas de cambará.
\end{abstract}

Palavras-chave: Espécie florestal nativa; Produção de mudas; Interação planta-micro-organismo.

\section{Trichoderma spp. IN EMERGENCE AND GROWTH OF CAMBARÁ SEEDLINGS (Gochnatia polymorpha (Less.) Cabrera)}

\begin{abstract}
In this work, the objective was to evaluate the effect of Trichoderma spp. isolates on the emergence of plantules and growth of cambará seedlings (Gochnatia polymorpha). For that we used, in a greenhouse, sterilized and unsterilized substrates, where we evaluated the effects of four isolates of trichoderma; TSM1 and TSM2 of Trichoderma viride, 2B2 and 2B22 of Trichoderma harzianum, plus a mix prepared with the mixture of these four isolates, and two commercial products based on trichoderma. Data analysis showed that trichoderma isolates tested did not interfere in plantule emergence, but the isolates $2 \mathrm{~B} 2$ and $2 \mathrm{~B} 22$ of T. harzinum present potentials as growth promoters of cambará seedlings.
\end{abstract}

Keywords: Native forest species; Seedling production; Plant-microorganism interaction.

\section{INTRODUÇÃO}

A ocupação do território brasileiro causou perdas do ponto de vista ambiental e florestal, sendo a obtenção de mudas florestais nativas para serem utilizadas em programas de reposição florestal, recuperação de áreas degradadas, arborização urbana, preservação de espécies nativas, além de outros interesses econômicos, um dos modos de contrabalancear a degradação gerada. A principal forma de propagação das árvores florestais nativas é por meio de sementes, no entanto as disponíveis em populações naturais não são suficientes para atender à demanda, além do que muitas dessas não apresentam qualidade adequada (SILVA; HIGA, 2006), o que dificulta a produção e o cultivo das mudas.

Gochnatia polymorpha é uma espécie arbórea nativa pertencente à família Asteraceae, com distribuição em vários estados brasileiros e diferentes denominações vulgares, como cambará-do-mato e candeia, em São Paulo, pau-candeia e cambarazinho no Paraná e cambará no Rio Grande do Sul. A espécie é recomendada para arborização urbana, recuperação de ecossistemas degradados e conservação do solo, sendo indicada

\footnotetext{
${ }^{1}$ Recebido em 28.06.2012 aceito para publicação em 09.12.2014.

${ }^{2}$ Universidade Federal de Santa Maria, Mestrado em Agrobiologia, Santa Maria, Rio Grande do Sul - Brasil. E-mail: $<$ danifmartins@gmail.com>.

${ }^{3}$ Universidade Federal de Santa Maria, Graduando em Agronomia, Santa Maria, Rio Grande do Sul - Brasil. E-mail: <antonio_apt88@yahoo.com.br>.

${ }^{4}$ Universidade Federal de Santa Maria, Departamento de Fitotecnia, UFSM, Santa Maria, Rio Grande do Sul - Brasil. E-mail: <sjlopes@pop.com.br>.

${ }^{5}$ Universidade Federal de Santa Maria, Centro de Ciências Naturais e Exatas, UFSM, Santa Maria, Rio Grande do Sul - Brasil. E-mail:<acfsilva2@uol.com.br>.
}

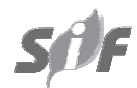

Revista Árvore, Viçosa-MG, v.39, n.1, p.167-176, 2015 http://dx.doi.org/10.1590/0100-67622015000100016 
como planta fixadora de barrancas de rios e reposição de mata ciliar. Além disso, proporciona madeira para obras imersas, construção civil e moirões; o tronco e as raízes produzem curvas para embarcações, carvão e lenha; apresenta características ornamentais e medicinais, sendo as folhas e cascas utilizadas no preparo de chás para afecções bronco-pulmonares; e as flores são melíferas (LORENZI, 1998; GLUFKE, 1999; CARVALHO, 2003; PIÑA-RODRIGUES et al., 2007).

A espécie produz, anualmente, grande quantidade de frutos do tipo cipsela, deiscente, oblongo, preto e sulcado, coroado de um papilho cerdoso e piloso, de 2 a 5 mm de comprimento (CARVALHO, 2003). A semente é inclusa no fruto e a coleta deste deve ser feita diretamente da árvore quando iniciar a queda espontânea. Um quilograma de sementes contém aproximadamente 2.200.000 unidades (LORENZI, 1998). De acordo com Carvalho (2003), a germinação das sementes é baixa, entre 30 e $50 \%$, e a falta de qualidade fisiológica de espécies florestais nativas pode ser, conforme Silva e Higa (2006), decorrente do pequeno número de árvores-matrizes disponíveis em populações naturais que se encontram extremamente fragmentadas. Além disso, sementes são estruturas atacadas por patógenos, o que afeta a qualidade e reduz sua capacidade germinativa.

Outro fator limitante na produção e cultivo de mudas florestais nativas é que algumas espécies demoram para iniciar a produção de sementes por apresentarem crescimento lento (CALDAS, 2006). Conforme Lorenzi (1998), o desenvolvimento de G. polymorpha no campo é rápido, podendo atingir $3 \mathrm{a} 4 \mathrm{~m}$ em dois anos, entretanto Carvalho (2003) mencionou que o crescimento é lento a moderado, com o crescimento em altura bastante expressivo até os 4 anos de idade.

As dificuldades enfrentadas no processo germinativo das sementes e no cultivo das mudas florestais nativas fazem que sejam comercializadas com baixa qualidade e alto custo. No entanto, algumas estratégias podem ser implantadas para amenizar esses problemas. O desenvolvimento de técnicas silviculturais para estimular a germinação de sementes e o crescimento vegetal é incipiente para a maioria das espécies florestais nativas, porém essas tecnologias poderiam beneficiar a produção de mudas (CALDAS, 2006).

A interação entre plantas e microrganismos é alternativa sustentável que vem sendo pesquisada e aplicada. Espécies de trichoderma estão entre os fungos mais estudados como agentes no controle biológico de fitopatógenos, na promoção da germinação de sementes e do crescimento vegetal (ALTOMARE et al., 1999). Diferentes isolados de trichoderma têm levado a aumentos significativos na porcentagem e precocidade de germinação, além de ocasionar aumento no crescimento e produtividade de culturas agrícolas inoculadas com esse bioagente, como tem sido observado em milho (Zea mays), feijão (Phaseolus vulgaris), ervilha (Pisum sativum), grão-de-bico (Cicer arietinum), pepino (Cucumis sativus), pimentão (Capsicum annum), rabanete (Raphanus sativus), tomate (Solanum lycopersicum), alface (Lactuca sativa), cenoura (Daucus carota) e algodão (Gossypium sp.), entre outras (MELO, 1998; YEDIDIA et al., 2001; HARMAN et al., 2004; RESENDE et al., 2004; JYOTSNA et al., 2008; HOYOS-CARVAJAL et al., 2009).

Apesar da inestimável contribuição de espécies de trichoderma em culturas agrícolas, poucos trabalhos têm sido realizados envolvendo esses fungos antagonistas e espécies florestais nativas (DONOSO et al., 2008), e os resultados dessa interação poderão otimizar a produção de mudas para os mais diversos interesses e, consequentemente, reduzir o extrativismo de florestas naturais; contribuir nos programas de reflorestamentos no Brasil, acelerando processos de recuperação de áreas degradadas; além da conservação genética das espécies e de benefícios para os produtores em viveiros. Assim, este trabalho objetivou avaliar o efeito de isolados de Trichoderma spp. na emergência de plântulas e no crescimento de mudas de cambará (Gochnatia polymorpha).

\section{MATERIAL E MÉTODOS}

Os ramos com infrutescências de G. polymorpha foram coletados em janeiro de 2011, em uma população natural, no Distrito Boca do Monte em Santa Maria,

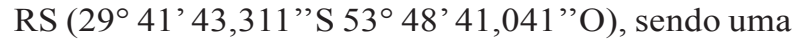
exsicata incorporada ao herbário do Departamento de Biologia (DB), do Centro de Ciências Naturais e Exatas (CCNE) da Universidade Federal de Santa Maria (UFSM), sob o número de registro SMDB - 13.139. Os frutos (diásporos) foram extraídos manualmente das infrutescências e mantidos em laboratório, à sombra, para secagem natural.

Foram realizados dois experimentos simultâneos em casa de vegetação do DB/CCNE/UFSM, com

Revista Árvore, Viçosa-MG, v.39, n.1, p.167-176, 2015

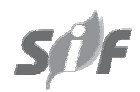


temperatura controlada a $25^{\circ} \mathrm{C}$. O substrato utilizado em ambos foi composto pela mistura de Tecnomax ${ }^{\circledR}$ e vermiculita expandida, na proporção 2:1. Em um dos experimentos, empregou-se substrato esterilizado e no outro, substrato não esterilizado. Em cada experimento, utilizaram-se 78 vasos com capacidade para $800 \mathrm{~mL}$, os quais foram preenchidos com $335 \mathrm{~g}$ de substrato. A esterilização do substrato foi efetuada em autoclave a $120^{\circ} \mathrm{C}$, durante $1 \mathrm{~h} \mathrm{e}$, decorridas $24 \mathrm{~h}$, mais uma hora de autoclavagem, visando eliminar a germinação de possíveis propágulos de resistência de microrganismos do substrato (ETHUR et al., 2005). Os vasos contendo substrato esterilizado foram mantidos em bancada separada daqueles com substrato não esterilizado.

Os isolados de trichoderma testados foram: TSM1 e TSM2 de Trichoderma viride, 2B2 e 2B22 de Trichoderma harzianum, que estavam armazenados no Laboratório de Interação Planta-Microrganismo/ CCNE/UFSM. Também, foi testado um mix, preparado com a mistura dos quatro referidos isolados, além de dois produtos comerciais à base de trichoderma: Agrotrich $^{\circledR}$ e Trichodermil ${ }^{\circledR}$, que foram adquiridos direto dos fabricantes. A aplicação dos isolados de trichoderma nos substratos foi através de pós-biológicos, preparados conforme descrição nos parágrafos subsequentes.

Para o preparo dos pós, discos de meio de cultura à base de aveia ( $40 \mathrm{~g}$ de aveia, $20 \mathrm{~g}$ de ágar e 1.000 $\mathrm{mL}$ de água destilada) contendo micélio e esporos dos isolados foram transferidos sobre $150 \mathrm{~g}$ de arroz umedecido com $25 \mathrm{~mL}$ de água destilada em sacos de polipropileno de $500 \mathrm{~mL}$, previamente esterilizados em autoclave a $120^{\circ} \mathrm{C}$ durante $1 \mathrm{~h}$ e, decorridas 24 $\mathrm{h}$, mais $1 \mathrm{~h}$ de autoclavagem. Esse material foi mantido a $25 \pm 3{ }^{\circ} \mathrm{C}$ e fotoperíodo de $12 \mathrm{~h}$ por 15 dias. Decorrido esse período, o arroz colonizado pelo fungo foi transferido para envelopes de papel $(25 \times 17 \mathrm{~cm})$, sendo realizada a secagem em estufa à temperatura de $35 \pm 3$ ${ }^{\circ} \mathrm{C}$, por cinco dias. Depois de seco, o arroz foi triturado e peneirado. Para estimar as unidades formadoras de colônias por grama de pó (UFC.g ${ }^{-1}$ ), procedeu-se á contagem usando a câmara de Neubauer e a técnica de diluição seriada (FORTES et al., 2007).

Realizou-se a introdução dos pós-biológicos nos substratos sete dias antes da semeadura da espécie florestal. Utilizaram-se $2 \mathrm{~g}$ de pó biológico por $\mathrm{kg}$ de substrato, conforme indicação dos fabricantes dos pós- comerciais, sendo essa dose ajustada para que os tratamentos recebessem o equivalente a $10^{6} \mathrm{UFC}^{-1} \mathrm{~g}^{-1} \mathrm{de}$ pó biológico, com exceção do Trichodermil ${ }^{\circledR}$, que possui $10^{8}$ UFC.g- ${ }^{-1}$. Utilizou-se menor concentração de esporos nos demais tratamentos, com o objetivo de verificar a superioridade de tais esporos quanto à capacidade de promoção de crescimento vegetal em relação ao único produto registrado no Ministério da Agricultura, Pecuária e Abastecimento (MAPA) (Trichodermil ${ }^{\circledR}$ ), na época da instalação do experimento. Os isolados TSM1, TSM2, 2B2, 2B22 e o mix também foram testados na dose de $4 \mathrm{~g}$ de pó por $\mathrm{kg}$ de substrato, além do tratamento-controle (sem trichoderma), em cada experimento. Foram semeados 50 diásporos, contendo uma semente cada, por vaso, à profundidade de $0,5 \mathrm{~cm}$. Adicionou-se água destilada nos vasos com o substrato até que este estivesse saturado, sendo essas quantidades de água destilada medidas e utilizadas para irrigação dos vasos, diariamente.

Após sete dias da semeadura, semanalmente foram contadas as plântulas emergidas até o final de 12 semanas, quando se calculou a porcentagem total de emergência, através do número de plântulas emergidas em relação ao número de diásporos colocados para germinar. Também, foi avaliada a primeira contagem de emergência, aos 28 dias, expressa em porcentagem; e o índice de velocidade de emergência (IVE), que foi calculado somando-se o número de plântulas emergidas a cada sete dias e dividido pelo respectivo número de dias transcorridos a partir da semeadura, conforme estabelecido por Maguire em 1962 (NAKAGAWA, 1994). A porcentagem de plântulas sobreviventes foi determinada no final das 12 semanas, através do número de plântulas sobreviventes em relação ao número de plântulas emergidas.

O efeito do trichoderma no crescimento das mudas foi verificado ao final das 12 semanas, quando foram realizados os seguintes procedimentos de avaliação: para o número de folhas, realizou-se a contagem por plântula, sendo calculada a média aritmética; a altura e o comprimento da maior raiz foram medidos em $\mathrm{cm}$, sendo calculada a média aritmética. Para avaliar a massa fresca da parte aérea e da raiz, as plântulas foram retiradas do substrato, lavadas em água corrente e, a seguir, deixadas para escorrer em papel absorvente. Em seguida, foi feito um corte na base do caule, separando-se a parte aérea da raiz, quando ocorreu a pesagem, em balança com precisão de $0,001 \mathrm{~g}$, da parte aérea e da raiz,

Revista Árvore, Viçosa-MG, v.39, n.1, p.167-176, 2015 
separadamente, e foi calculada a média aritmética por plântula. Após ter-se obtido a massa fresca, o material foi colocado em sacos de papel e levado para estufa, mantido à temperatura de $60-65^{\circ} \mathrm{C}$, assim permanecendo até atingir massa constante, quando foi pesado e a média de massa seca da parte aérea e da raiz por plântula determinada (NAKAGAWA, 1994).

Foram avaliados os seguintes tratamentos em cada experimento: 1) TSM1 dose 1;2) TSM1 dose 2; 3) TSM2 dose 1 ; 4) TSM2 dose 2; 5) 2B2 dose 1; 6) 2B2 dose 2; 7) 2B22 dose 1;8) 2B22 dose 2;9) mix dos quatro isolados de trichoderrma dose 1 ; 10) mix dos quatro isolados de trichoderma dose 2 ; 11) Trichodermil ${ }^{\mathbb{R}}$ dose 1 ; 12) Agrotrich ${ }^{\circledR}$ dose 1 ; e 13) tratamento-controle (sem trichoderma). Optou-se por utilizar substrato esterilizado e não esterilizado, a fim de fazer um estudo da ação dos isolados de trichoderma nos dois ambientes. Nos dois experimentos, os tratamentos foram dispostos em delineamento inteiramente casualizado, com seis repetições por tratamento; cada parcela experimental foi constituída por um vaso contendo 50 diásporos, num total de 300 por tratamento.

Os dados em porcentagem foram transformados por arcosseno da raiz quadrada e os demais, transformados pelo procedimento Box Cox. Em seguida, foram submetidos à análise de variância (ANOVA) e as médias, comparadas pelo teste de Tukey a 5\% de probabilidade de erro, pelo programa estatístico Assistat 7.6 beta (SILVA; AZEVEDO, 2009). Os dados que não atenderam às pressuposições da ANOVA foram submetidos ao teste de Kruskal-Wallis pelo programaAction 2.2 (PORTAL ACTION, 2011).

\section{RESULTADOS}

Em relação aos resultados das variáveis emergência e sobrevivência das plântulas, tanto em substrato esterilizado quanto em não esterilizado, não houve diferença significativa entre os tratamentos com a introdução dos isolados de trichoderma e o tratamentocontrole sem trichoderma (dados não apresentados nas Tabelas 1 e 2).

Quanto à análise de primeira contagem e índice de velocidade de emergência das plântulas, no substrato esterilizado, observou-se que T. harzianum isolado 2B2 dose 1 foi superior ao controle. No substrato não esterilizado, não houve influência dos isolados de trichoderma para as referidas variáveis.

Revista Árvore, Viçosa-MG, v.39, n.1, p.167-176, 2015
A análise das avaliações das plântulas quanto ao número de folhas demonstrou que os isolados 2B2 e 2B22 de T. harzianumdos e 2 apresentaram as maiores médias em substrato esterilizado. $\mathrm{O}$ tratamento com o isolado 2B22 dose 2 também indicou o melhor resultado quanto à altura das plântulas. No substrato não esterilizado, o isolado 2B22 apresentou resultado satisfatório para essas variáveis na dose 2 .

Quanto ao comprimento da maior raiz, G. polymorpha apresentou bom crescimento mesmo sem a adição de trichoderma. No substrato esterilizado, o tratamento com resultado satisfatório em relação ao controle foi aquele constituído pelo isolado $2 \mathrm{~B} 2$ na dose 2 , mas a média desse tratamento não diferiu dos tratamentos com os isolados TSM1 dose 2, 2B2 dose 1 e 2B22 dose 1. No substrato não esterilizado, para essa mesma variável, somente o tratamento com o isolado 2B22 dose 2 diferiu do tratamento-controle, mas não diferiu das médias dos tratamentos TSM2 dose 1, 2B2 doses 1 e 2, 2 B22 dose 1 e mix dose 2 , que não diferiram do tratamentocontrole.

No substrato esterilizado, o isolado que proporcionou a maior média de massa fresca da parte aérea foi o 2B22 na dose 2, diferindo do controle, mas não do tratamento $2 \mathrm{~B} 2$ dose 2 . O isolado que proporcionou a maior média de massa fresca da raiz foi o $2 \mathrm{~B} 2$ dose 2 , que diferiu do controle, mas não dos tratamentos 2B2 dose 1 e 2B22 doses 1 e 2 . O isolado que proporcionou a maior média de massa seca de parte aérea e raiz foi o 2B22 dose 2, que diferiu do controle, mas para a massa seca da parte aérea não diferiu do $2 \mathrm{~B} 2$ dose 2 , enquanto para massa seca da raiz não diferiu do $2 \mathrm{~B} 2$ dose $2 \mathrm{e}$ 2B22 dose 1. Nas análises do substrato não esterilizado, o isolado 2B22 dose 2 também apresentou maior média de massa fresca da parte aérea e da raiz, diferindo do controle, mas não do TSM2 dose 1, 2B2 doses 1 e 2, 2B22 dose 1, mix dose 2, Trichodermil ${ }^{\circledR}$ e Agrotrich ${ }^{\circledR}$. Em relação à massa seca, tanto de parte aérea quanto de raiz, não houve diferença significativa entre os tratamentos.

\section{DISCUSSÃO}

Os mecanismos de ação dos fungos promotores do crescimento vegetal são específicos e podem variar conforme o ambiente, como substrato, disponibilidade de nutrientes e interferência de outros microrganismos. Nesse sentido, os tratamentos avaliados no substrato 
Tabela 1 - Efeito de isolados de trichoderma na primeira contagem de emergência (PC), índice de velocidade de emergência (IVE), número de folhas, altura, comprimento da maior raiz, massa fresca (MF) da parte aérea (PA) e da raiz e massa seca (MS) da parte aérea e da raiz de Gochnatia polymorpha em substrato esterilizado. Santa Maria, RS (2011).

Table 1 - Effect of trichoderma isolates in the first count of emergence (FC), emergence speed index (ESI), leaf number, height, length of the longest root, fresh weight (FW) of aerial part (AP) and root and dry weight (DW) of aerial part and root of Gochnatia polymorpha in sterilized substrate. Santa Maria, RS (2011).

\begin{tabular}{|c|c|c|c|c|c|c|c|c|c|}
\hline Tratamentos & $\mathrm{PC}(\%)$ & IVE & $\begin{array}{l}\text { Número } \\
\text { de folhas }\end{array}$ & $\begin{array}{l}\text { Altura } \\
(\mathrm{cm})\end{array}$ & $\begin{array}{l}\text { Comprimento } \\
\text { raiz }(\mathrm{cm})\end{array}$ & $\begin{array}{c}\text { MF PA } \\
(\mathrm{g})\end{array}$ & $\begin{array}{c}\text { MF raiz } \\
\text { (g) }\end{array}$ & $\begin{array}{c}\text { MS PA } \\
(\mathrm{g})\end{array}$ & $\begin{array}{c}\text { MS raiz } \\
\text { (g) }\end{array}$ \\
\hline 1.TSM1 (1) & $5,67 \mathrm{bc}^{*}$ & $0,210 \mathrm{bc}$ & $3,64 \mathrm{bcd}$ & $1,05 \mathrm{~cd}$ & $10,19 \mathrm{~d}$ & 0,082 def & $0,091 \mathrm{efg}$ & 0,022 ef & $0,019 \mathrm{efg}$ \\
\hline 2. TSM1(2) & $10,33 \mathrm{abc}$ & $0,338 \mathrm{abc}$ & $3,82 \mathrm{bcd}$ & $1,06 \mathrm{~cd}$ & $11,86 \mathrm{abcd}$ & 0,102 cde & $0,111 \mathrm{cdef}$ & $0,027 \mathrm{cdef}$ & $0,023 \mathrm{cdef}$ \\
\hline 3. TSM2 (1) & $3,67 \mathrm{c}$ & $0,193 \mathrm{bc}$ & $3,22 \mathrm{~cd}$ & $0,81 \mathrm{de}$ & $9,82 \mathrm{~d}$ & $0,046 \mathrm{def}$ & $0,048 \mathrm{fg}$ & $0,012 \mathrm{efg}$ & $0,010 \mathrm{fg}$ \\
\hline 4. TSM2 (2) & $7,67 \mathrm{abc}$ & $0,276 \mathrm{abc}$ & $3,80 \mathrm{bcd}$ & $0,96 \mathrm{cde}$ & $10,79 \mathrm{~cd}$ & $0,073 \mathrm{def}$ & 0,090 defg & $0,018 \mathrm{def}$ & $0,018 \mathrm{defg}$ \\
\hline 5. 2B2 (1) & $16,67 \mathrm{a}$ & $0,475 \mathrm{a}$ & $4,71 \mathrm{abc}$ & $1,55 \mathrm{bc}$ & $16,47 \mathrm{abc}$ & $0,281 \mathrm{c}$ & 0,319 abcd & $0,073 \mathrm{~cd}$ & $0,055 \mathrm{bcd}$ \\
\hline 6. 2B2 (2) & $14,67 \mathrm{ab}$ & $0,370 \mathrm{abc}$ & $6,75 \mathrm{a}$ & $2,69 \mathrm{ab}$ & $18,26 \mathrm{a}$ & $0,916 \mathrm{ab}$ & $0,875 \mathrm{a}$ & $0,249 \mathrm{ab}$ & $0,138 \mathrm{ab}$ \\
\hline 7. $2 \mathrm{~B} 22(1)$ & $9,00 \mathrm{abc}$ & $0,282 \mathrm{abc}$ & $5,00 \mathrm{ab}$ & $1,50 \mathrm{abc}$ & $13,92 \mathrm{abcd}$ & $0,273 \mathrm{bc}$ & $0,314 \mathrm{abc}$ & $0,072 \mathrm{bc}$ & $0,056 \mathrm{abc}$ \\
\hline 8. $2 \mathrm{~B} 22(2)$ & $16,00 \mathrm{ab}$ & $0,414 \mathrm{ab}$ & $7,36 \mathrm{a}$ & $3,60 \mathrm{a}$ & $16,98 \mathrm{ab}$ & $1,113 \mathrm{a}$ & $0,895 \mathrm{ab}$ & $0,317 \mathrm{a}$ & $0,154 \mathrm{a}$ \\
\hline 9. MIX (1) & $11,67 \mathrm{abc}$ & $0,341 \mathrm{abc}$ & $2,60 \mathrm{~d}$ & $0,68 \mathrm{e}$ & $9,57 \mathrm{~d}$ & $0,022 \mathrm{f}$ & $0,030 \mathrm{~g}$ & $0,004 \mathrm{~g}$ & $0,006 \mathrm{~g}$ \\
\hline 10. MIX (2) & $3,67 \mathrm{c}$ & $0,154 \mathrm{c}$ & $2,80 \mathrm{~d}$ & $0,78 \mathrm{de}$ & $8,74 \mathrm{~d}$ & 0,037 ef & $0,043 \mathrm{fg}$ & $0,009 \mathrm{fg}$ & $0,008 \mathrm{fg}$ \\
\hline 11. Trichodermil ${ }^{\mathbb{E}}(1)$ & $3,67 \mathrm{c}$ & $0,177 \mathrm{c}$ & $2,90 \mathrm{~d}$ & $0,77 \mathrm{de}$ & $10,02 \mathrm{~d}$ & $0,061 \mathrm{def}$ & $0,060 \mathrm{efg}$ & $0,015 \mathrm{efg}$ & $0,012 \mathrm{efg}$ \\
\hline 12. Agrotrich $^{\circledR}(1)$ & $6,67 \mathrm{abc}$ & $0,243 \mathrm{bc}$ & $3,46 \mathrm{bcd}$ & $1,03 \mathrm{~cd}$ & $11,62 \mathrm{bcd}$ & $0,137 \mathrm{~cd}$ & 0,192 bcde & 0,034 cde & 0,032 cde \\
\hline 13. Controle & $3,00 \mathrm{c}$ & $0,166 \mathrm{c}$ & $2,67 \mathrm{~d}$ & $0,80 \mathrm{de}$ & $10,70 \mathrm{bcd}$ & 0,065 def & 0,079 efg & 0,014 efg & $0,013 \mathrm{efg}$ \\
\hline Média & 8,64 & 0,279 & 4,06 & 1,33 & 12,23 & 0,246 & 0,242 & 0,067 & 0,042 \\
\hline $\mathrm{CV} \%$ & 37,14 & 40,19 & 11,78 & 12,26 & 15,98 & 21,67 & 19,80 & 22,33 & 22,41 \\
\hline
\end{tabular}

*Médias não seguidas pela mesma letra diferem significativamente entre si, pelo teste Tukey a 5\% de probabilidade de erro.

Nos tratamentos com (1) e (2), lê-se dose 1 e dose 2 . Dose $1=2 \mathrm{gkg}^{-1}$ de substrato $\left(10^{6} \mathrm{UFCg}^{-1}\right.$ de pó biológico); e dose $2=4$ gkg ${ }^{1}$ de substrato $\left(10^{6} \mathrm{UFCg}^{-1}\right.$ de pó biológico)

Trichodermil $1^{\circledR}=10^{8} \mathrm{UFC}^{-1}$ de pó biológico.

Tabela 2 - Efeito de isolados de trichoderma na primeira contagem de emergência (PC), índice de velocidade de emergência (IVE), número de folhas, altura, comprimento da maior raiz, massa fresca (MF) de parte aérea (PA) e de raiz e massa seca (MS) de parte aérea e de raiz de Gochnatia polymorpha em substrato não esterilizado. Santa Maria, RS (2011).

Table 2 - Effect of trichoderma isolates in the first count of emergence (FC), emergence speed index (ESI), leaf number, height, length of the longest root, fresh weight (FW) of aerial part (AP) and root and dry weight (DW) of aerial part and root of Gochnatia polymorpha in unsterilized substrate. Santa Maria, RS (2011).

\begin{tabular}{|c|c|c|c|c|c|c|c|c|c|}
\hline Tratamentos & PC (\%) & IVE & $\begin{array}{l}\text { Número } \\
\text { de folhas }\end{array}$ & $\begin{array}{l}\text { Altura } \\
(\mathrm{cm})\end{array}$ & $\begin{array}{c}\text { Comprimento } \\
\text { raiz }(\mathrm{cm})\end{array}$ & $\begin{array}{c}\text { MF PA } \\
(\mathrm{g})\end{array}$ & $\begin{array}{c}\text { MF raiz } \\
\text { (g) }\end{array}$ & $\begin{array}{c}\text { MS PA } \\
\text { (g) }\end{array}$ & $\begin{array}{c}\text { MS raiz } \\
\text { (g) }\end{array}$ \\
\hline 1.TSM1 (1) & $2,00 \mathrm{ab}^{*}$ & $0,108 \mathrm{ab}$ & $2,40 \mathrm{c}$ & $0,63 \mathrm{c}$ & $7,79 \mathrm{~b}$ & $0,023 \mathrm{bc}$ & $0,029 \mathrm{bc}$ & $0,006 \mathrm{bc}$ & $0,007 \mathrm{abc}$ \\
\hline 2. TSM1 (2) & $1,00 \mathrm{ab}$ & $0,094 \mathrm{ab}$ & $2,69 \mathrm{c}$ & $0,62 \mathrm{c}$ & $6,61 \mathrm{~b}$ & $0,017 \mathrm{c}$ & $0,018 \mathrm{c}$ & $0,004 \mathrm{bc}$ & $0,004 \mathrm{c}$ \\
\hline 3. TSM2 (1) & $2,00 \mathrm{ab}$ & $0,164 \mathrm{ab}$ & $2,77 \mathrm{c}$ & $0,74 \mathrm{c}$ & $9,27 \mathrm{ab}$ & $0,035 \mathrm{abc}$ & $0,042 a b c$ & $0,007 \mathrm{abc}$ & $0,007 \mathrm{abc}$ \\
\hline 4. TSM2 (2) & $1,33 \mathrm{ab}$ & $0,084 \mathrm{~b}$ & $2,68 \mathrm{c}$ & $0,75 \mathrm{c}$ & $8,52 \mathrm{~b}$ & $0,026 \mathrm{bc}$ & $0,030 \mathrm{bc}$ & $0,006 \mathrm{bc}$ & $0,006 \mathrm{bc}$ \\
\hline 5. 2B2 (1) & $4,00 \mathrm{ab}$ & $0,157 \mathrm{ab}$ & $3,58 \mathrm{bc}$ & $0,86 \mathrm{bc}$ & $11,74 \mathrm{ab}$ & $0,063 \mathrm{abc}$ & $0,095 \mathrm{abc}$ & $0,015 \mathrm{abc}$ & $0,018 \mathrm{abc}$ \\
\hline 6. 2B2 (2) & $4,67 \mathrm{ab}$ & $0,206 \mathrm{ab}$ & $4,76 \mathrm{ab}$ & $1,34 \mathrm{ab}$ & $13,22 \mathrm{ab}$ & $0,250 \mathrm{ab}$ & $0,295 \mathrm{ab}$ & $0,059 \mathrm{ab}$ & $0,046 \mathrm{ab}$ \\
\hline 7. 2B22 (1) & $3,00 \mathrm{ab}$ & $0,165 \mathrm{ab}$ & $3,33 \mathrm{bc}$ & $0,83 \mathrm{bc}$ & $13,04 \mathrm{ab}$ & $0,068 \mathrm{abc}$ & $0,105 \mathrm{abc}$ & $0,015 \mathrm{abc}$ & $0,018 \mathrm{abc}$ \\
\hline 8. 2B22 (2) & 7,33 a & $0,244 \mathrm{a}$ & $5,94 \mathrm{a}$ & $1,94 \mathrm{a}$ & $18,19 \mathrm{a}$ & $0,574 \mathrm{a}$ & $0,650 \mathrm{a}$ & $0,152 \mathrm{a}$ & $0,108 \mathrm{a}$ \\
\hline 9. MIX (1) & $4,33 \mathrm{ab}$ & $0,195 \mathrm{ab}$ & $2,56 \mathrm{c}$ & $0,67 \mathrm{c}$ & $8,26 \mathrm{~b}$ & $0,019 \mathrm{bc}$ & $0,022 \mathrm{bc}$ & $0,004 \mathrm{c}$ & $0,004 \mathrm{bc}$ \\
\hline 10. MIX (2) & $0,33 \mathrm{~b}$ & $0,071 \mathrm{~b}$ & $2,79 \mathrm{c}$ & $0,75 \mathrm{c}$ & $8,66 \mathrm{ab}$ & $0,027 \mathrm{abc}$ & $0,028 \mathrm{abc}$ & $0,005 \mathrm{abc}$ & $0,005 \mathrm{bc}$ \\
\hline 11. Trichodermil $®(1)$ & $3,33 \mathrm{ab}$ & $0,138 \mathrm{ab}$ & $2,72 \mathrm{c}$ & $0,68 \mathrm{c}$ & $8,55 \mathrm{~b}$ & $0,029 \mathrm{abc}$ & $0,037 \mathrm{abc}$ & $0,007 \mathrm{bc}$ & $0,008 \mathrm{bc}$ \\
\hline 12. Agrotricho $®(1)$ & $2,33 \mathrm{ab}$ & $0,114 \mathrm{ab}$ & $2,49 \mathrm{c}$ & $0,72 \mathrm{c}$ & $8,86 \mathrm{~b}$ & $0,030 \mathrm{abc}$ & $0,040 \mathrm{abc}$ & $0,006 \mathrm{abc}$ & $0,008 \mathrm{abc}$ \\
\hline 13. Controle & $2,67 \mathrm{ab}$ & $0,137 \mathrm{ab}$ & $2,60 \mathrm{c}$ & $0,73 \mathrm{c}$ & $9,52 \mathrm{~b}$ & $0,022 \mathrm{bc}$ & $0,027 \mathrm{bc}$ & $0,005 \mathrm{abc}$ & $0,006 \mathrm{abc}$ \\
\hline Média & 2,95 & 0,144 & 3,18 & 0,87 & 10,17 & 0,091 & 0,109 & 0,022 & 0,019 \\
\hline $\mathrm{CV} \%$ & - & 27,61 & 22,32 & 18,51 & 17,34 & - & - & - & - \\
\hline
\end{tabular}

* Médias não seguidas pela mesma letra diferem significativamente entre si, pelo teste Tukey a $5 \%$ de probabilidade de erro.

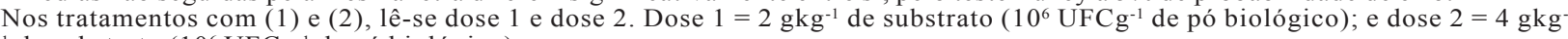

${ }^{1}$ de substrato $\left(10^{6} \mathrm{UFCg}^{-1}\right.$ de pó biológico).

Trichodermil ${ }^{\circledR}=10^{8} \mathrm{UFC} \mathrm{g}^{-1}$ de pó biológico 
esterilizado também foram avaliados em substrato não esterilizado, a fim de se conhecer a resposta dos isolados de trichoderma nos dois ambientes.

O efeito dos isolados de trichoderma observado nas diferentes variáveis analisadas vai ao encontro de Melo (1998), que afirmou que o sucesso da promoção de crescimento vegetal por bioagentes depende das propriedades e mecanismos de ação do organismo. Segundo esse autor, ao introduzir antagonistas no solo pode ocorrer hiperparasitismo entre espécies diferentes de trichoderma, e a introdução de dado isolado no solo pode, ainda, sofrer interferência de outras espécies indígenas, acarretando efeitos diferenciados.

Os resultados evidenciaram que os isolados de trichoderma testados não promoveram a emergência das plântulas de G. polymorpha. Resultados opostos foram encontrados por Kleifeld e Chet (1992), que observaram a ação positiva de Trichoderma harzianum em tratamento de semente e de solo na emergência de plântulas de feijão (Phaseolus vulgaris), rabanete (Raphanus sativus), tomate (Solanum lycopersicum) e pepino (Cucumis sativus). Luz (2001) verificou aumento significativo na emergência de plântulas de milho (Zea mays) através da microbiolização com T. harzianum isolado T-22, e Diniz et al. (2006) observaram que a inoculação das sementes de alface (Lactuca sativa) com Trichoderma viride promoveu aumento na emergência das plântulas. Já Ousley et al. (1993) constataram que alguns isolados auxiliaram e outros inibiram a germinação de sementes de alface (L. sativa). Esses resultados indicaram que o mecanismo de promoção de germinação é específico, e alguns isolados podem, ainda, inibir a germinação, o que não foi observado neste estudo.

$\mathrm{Na}$ análise de primeira contagem de emergência, em substrato esterilizado, verificou-se que T. harzianum isolado 2B2 dose 1 foi superior ao controle e acelerou a velocidade de emergência. Já em substrato não esterilizado, com essas mesmas variáveis, não se verificou efeito dos isolados de trichoderma sobre a emergência de plântulas de G. polymorpha. Esses resultados demonstraram que para o substrato esterilizado, embora na porcentagem total de emergência não tenha ocorrido diferença significativa, o tratamento com trichoderma proporcionou maior número de plântulas emergidas em menor número de dias, possivelmente por permitir que essas plântulas não fossem atacadas por patógenos habitantes da espermosfera, que poderiam afetar a emergência e estabelecimento das plantinhas. De acordo com Melo (1998), a aplicação de trichoderma não proporciona aumentos significativos somente na porcentagem, mas também na precocidade e velocidade de germinação.

Os resultados s correspondem aos encontrados por Oliveira (2007) em sementes de cártamo (Carthamus tinctorius), espécie também pertencente à família Asteraceae, em que o isolado TC 1.15 de Trichoderma sp. permitiu maior média de plântulas emergidas, mas que não diferiu dos outros tratamentos; no entanto, proporcionou o maior IVE, com diferença significativa. Ozbay et al. (2004) observaram que os isolados T22 e T95 de T. harzianum não auxiliaram na emergência do tamateiro, mas aumentaram o crescimento das plântulas.

Fortes et al. (2007) concluíram que os isolados ST, E15 e S2 de Trichoderma sp. aumentaram a sobrevivência de microestacas de eucalipto (Eucalyptus sp.). Resultados opostos foram obtidos neste estudo, em que os isolados de trichoderma testados não favoreceram as médias de sobrevivência das plântulas. Todavia, a promoção do crescimento vegetativo de G. polymorpha pôde ser verificada, em substrato esterilizado, pelas análises do número de folhas, altura, comprimento da maior raiz, massa fresca e seca da parte aérea e da raiz, em que foram observados efeitos significativos dos isolados de trichoderma. Segundo Contreras-Cornejo et al. (2009), a aplicação de trichoderma tem proporcionado além de aumentos na porcentagem e precocidade de germinação, assim como no peso de matéria seca, na altura das plântulas e no desenvolvimento das raízes laterais.

Quanto ao número de folhas, observou-se, em substrato esterilizado, que os isolados 2B2 e 2B22 na dose 2 apresentaram melhores resultados em relação ao controle, já na variável altura de plântula somente o isolado 2B22, na mesma dose, foi superior ao controle. Em substrato não esterilizado, o tratamento 2B22 dose 2 também foi o que teve melhores resultados para as referidas variáveis, em relação ao controle. Inbar et al. (1994), estudando o efeito de trichoderma no crescimento de pepineiro, observaram aumento de área foliar em plântulas cultivadas em substrato tratado 
com o isolado T-203 de T. harzianum. Filho et al. (2008) observaram diferença significativa na altura de mudas de eucalipto (Eucalyptus grandis x Eucalyptus urophylla) e de Eucalyptus camadulensis por isolados de Trichoderma sp. Donoso et al. (2008) concluíram que $T$. harzianum promoveu a maior média de altura em plântulas de Pinus radiata.

As mudas de G. polymorpha apresentaram bom crescimento da raiz principal, mas o isolado $2 \mathrm{~B} 2$ dose 2 , no substrato esterilizado, e o isolado $2 \mathrm{~B} 22$ dose 2 , no substrato não esterilizado, exibiram os melhores resultados, em comparação com o tratamento-controle. Harman (2000) também obteve maior crescimento de raízes de soja (Glycine max) e de milho (Z. mays) tratadas com $T$. harzianum T-22 quando comparados com testemunhas não tratados. Fortes et al. (2007) observaram que o isolado E15 promoveu o enraizamento de microestacas de eucalipto (Eucalyptus sp.), apresentando aumento significativo na porcentagem de enraizamento.

Em substrato esterilizado, os resultados indicaram efeito positivo do isolado $2 \mathrm{~B} 22$ dose 2 para a massa fresca da parte aérea, bem como para a massa seca tanto da parte aérea quanto da raiz. Para a variável massa fresca da raiz, o tratamento que obteve melhor resultado comparado ao controle foi o $2 \mathrm{~B} 2$ dose 2 . Em substrato não esterilizado, o isolado $2 \mathrm{~B} 22$ dose 2 foi superior quanto às variáveis massa fresca da parte aérea e da raiz, no entanto nenhum isolado apresentou efeito sobre a massa seca dessas variáveis.

Segundo Ethur et al. (2005), o comportamento de fungos de solo, como trichoderma, pode se modificar quando colocado em outro ambiente. O processo de autoclavagem pode ter acelerado a decomposição da matéria orgânica, causando modificações na solubilidade ou disponibilidade dos nutrientes. Conforme Kleifeld e Chet (1992), a habilidade de T. harzianum em promover o crescimento de plantas varia com o tipo de substrato, como nutrientes ou matérias orgânicas disponíveis e com a habilidade de competir com patógenos na rizosfera.

Tavares (2009) também obteve aumento na biomassa fresca e seca total de plantas de mamoeiro plantadas em solo contendo T. harzianum isolado T70 e Trichoderma virens isolado T68. Resende et al. (2004) observaram que T. harzianum estimulou maior acúmulo de matéria seca em plântulas de milho
(Z. mays). Filho et al. (2008), avaliando a promoção de crescimento de mudas de um clone híbrido de eucalipto (Eucalyptus grandi x E. urophylla), também observaram que o isolado CEN 162 de Trichoderma asperellum e o isolado CEN 262 de T. harzianum tiveram as maiores médias de massa seca da raiz e da parte aérea.

A propriedade de promoção do crescimento por trichoderma pode estar relacionada com o controle de fungos fitopatogênicos, através, principalmente, das ações de competição, parasitismo direto e antibiose (MELO, 1998), mas também envolve a disponibilização de nutrientes do solo e aumento na captação e translocação de minerais pelo desenvolvimento das raízes da planta (KLEIFELD; CHET, 1992). Segundo Altomare et al. (1999), no solo, macro e micronutrientes sofrem equilíbrio dinâmico de solubilização e insolubilização influenciado pela microflora, que afeta a acessibilidade destes para serem absorvidos pelas raízes das plantas. Conforme esses autores, o crescimento vegetal promovido por $T$. harzianum está na sua habilidade de solubilizar muitos nutrientes importantes para a planta e de produzir hormônios ou outros fatores de crescimento.

Carvalho Filho et al. (2008) observaram produção do hormônio ácido indolacético (AIA) em alguns isolados de Trichoderma spp., sendo em baixos níveis nos isolados CEN 209 e CEN 500 e níveis consideravelmente superiores no isolado CEN 262. Hoyos-Carvajal et al. (2009) avaliaram a produção de metabólitos de 101 isolados de Trichoderma spp., em que $60 \%$ das cepas produziram ácido indolacético (AIA) ou análogos à auxina, 20\% foram capazes de produzir formas solúveis de fosfato de rocha fosfática e $8 \%$ mostraram capacidade de produzir sideróforos consistentes para converter ferro a formas solúveis.

Sob essa perspectiva, verificou-se que os isolados 2B2 e 2B22 de T. harzianum apresentaram-se potenciais como promotores do crescimento vegetal para algumas das variáveis analisadas em plântulas de G. polymorpha (Figuras 1 e 2). Considerando a importância econômica e ambiental de espécies florestais nativas, esses resultados são relevantes para o aprimoramento de técnicas silviculturais relacionadas à realização de outros estudos que envolvam a promoção de crescimento de mudas florestais por bioagentes, como o trichoderma.

Revista Árvore, Viçosa-MG, v.39, n.1, p.167-176, 2015 


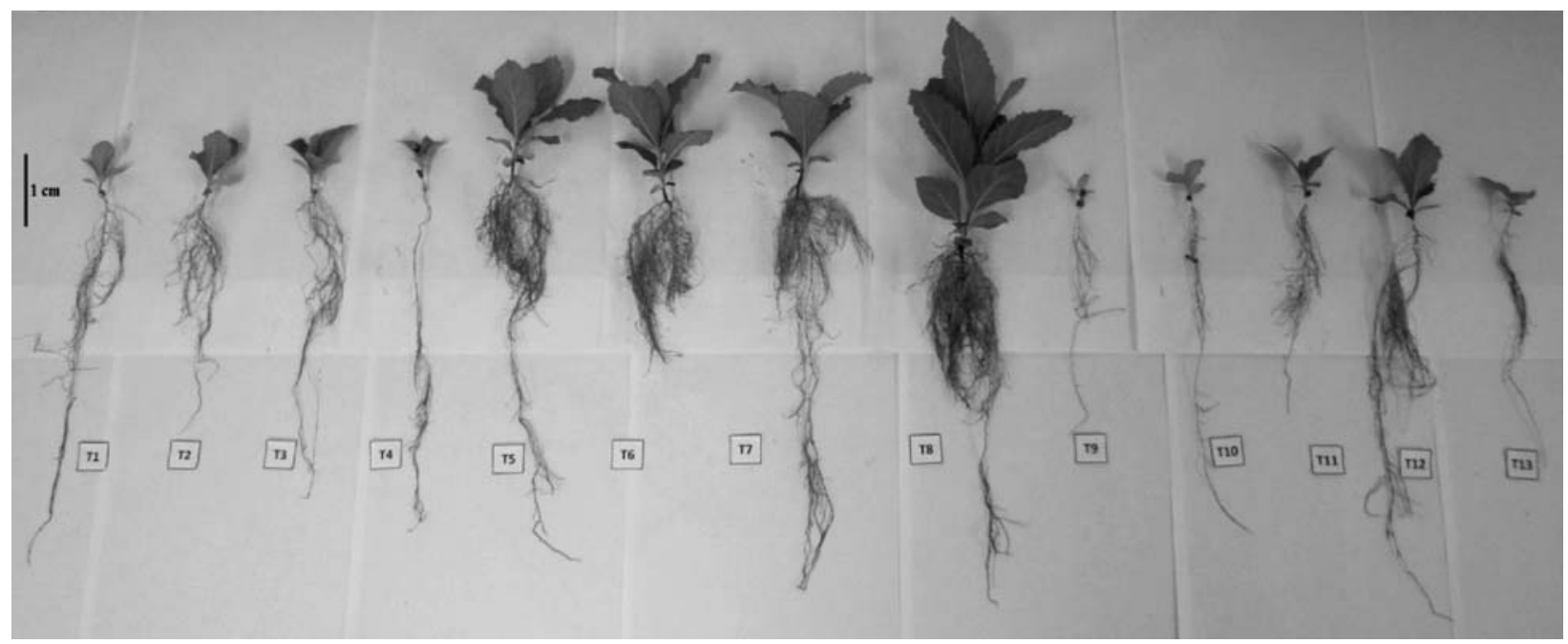

Figura 1 - Diferenças no crescimento vegetal de plântulas de Gochnatia polymorpha em função de isolados de trichoderma e do tratamento-controle em substrato esterilizado. T1: TSM1 dose 1; T2: TSM1 dose 2; T3: TSM2 dose 1; T4: TSM2 dose 2; T5: 2B2 dose 1; T6: $2 \mathrm{~B} 2$ dose 2; T7: 2B22 dose 1; T8: 2B22 dose 2; T9: mix dose 1; T10: mix dose 2; T11: Trichodermil ${ }^{\circledR}$ dose 1; T12: Agrotrich $^{\circledR}$ dose 1; e T13: controle (sem trichoderma).

Figure 1 -Differences in plant growth of plantules of Gochnatia polymorpha in function of trichoderma isolates and control treatment in sterilized substrate. T1: TSM1 dose 1; T2: TSM1 dose 2; T3: TSM2 dose 1; T4: TSM2 dose 2; T5: 2 B2 dose 1; T6: 2 B2 dose 2; T7: 2 B22 dose 1; T8: 2 B22 dose 2; T9: mix dose 1; T10: mix dose 2; T11: Trichodermil ${ }^{\circledR}$ dose 1; T12: Agrotrich ${ }^{\circledR}$ dose 1; and T13: control (no trichoderma).

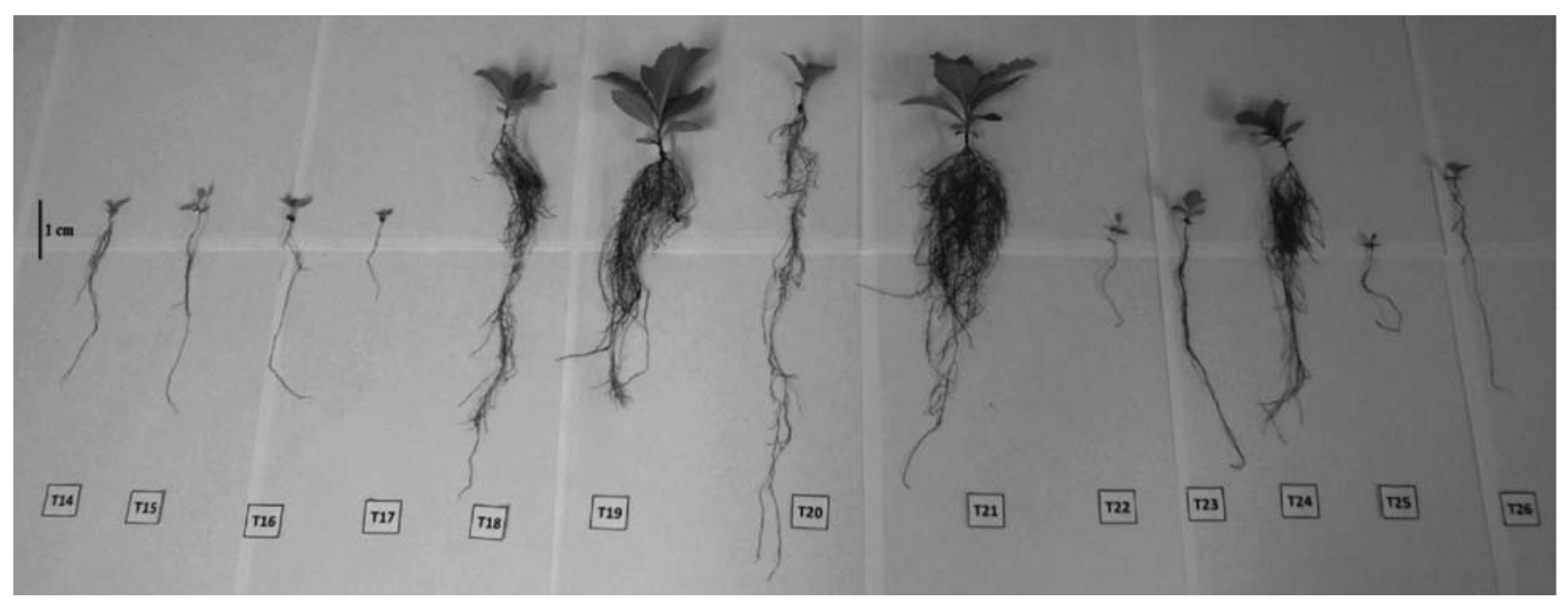

Figura 2 - Diferenças no crescimento vegetal de plântulas de Gochnatia polymorpha em função de isolados de trichoderma e do tratamento-controle em substrato não esterilizado. T14: TSM1 dose 1; T15: TSM1 dose 2; T16: TSM2 dose 1; T17: TSM2 dose 2; T18: 2B2 dose 1; T19: 2B2 dose 2; T20: 2B22 dose 1; T21: $2 \mathrm{~B} 22$ dose 2; T22: mix dose 1; T23: mix dose 2; T24: Trichodermil $^{\circledR}$ dose 1; T25: Agrotrich $^{\circledR}$ dose 1; e T26: controle (sem trichoderma).

Figure 2 - Differences in plant growth of plantules of Gochnatia polymorpha in function of trichoderma isolates and control treatment in unsterilized substrate. T14: TSM1 dose 1; T15: TSM1 dose 2; T16: TSM2 dose 1; T17: TSM2 dose 2; T18: 2 B2 dose 1; T19: 2B2 dose 2; T20: 2 B22 dose 1; T21: 2 B22 dose 2; T22: mix dose 1; T23: mix dose

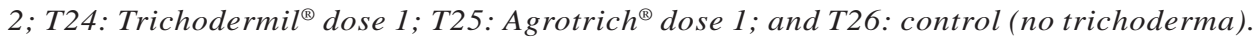

Revista Árvore, Viçosa-MG, v.39, n.1, p.167-176, 2015 


\section{CONCLUSÃO}

Os isolados de trichoderma testados não interferem na emergência das plântulas, mas os isolados 2B2 e 2B22 de T. harzianum apresentam potenciais como promotores do crescimento vegetal de mudas de cambará (G. polymorpha).

\section{AGRADECIMENTOS}

À Coordenação de Aperfeiçoamento de Pessoal de Nível Superior (CAPES), pela bolsa de mestrado concedida ao primeiro autor.

\section{REFERÊNCIAS}

ALTOMARE, C.; NORVELL, W. A.; BJORKMAN, T.; HARMAN, G. E. Solubilization of phosphates and micronutrients by the plant-growth-promoting and biocontrol fungus Trichoderma harzianum Rifai 1295-22. Applied and Environmental Microbiology, v.65, n.7, p.2926-2933, 1999.

CALDAS, L. S. Pomares de sementes de espécies nativas as funções das redes de sementes. In: HIGA, A. R.; Silva, L. D. (Coord.). Pomar de sementes de espécies florestais nativas. Curitiba: FUPEF, 2006. p.227-241.

CARVALHO, P. E. R. Espécies arbóreas brasileiras. Brasília: Embrapa, 2003. v.1.p.274280.

CONTRERAS-CORNEJO, H. A.; MACÍASRODRÍGUES, L.; CORTÉS-PENAGOS, C.; LÓPEZBUCIO, J. Trichoderma virens, a plant beneficial fungus, enhances biomass production and promotes lateral root growth through an auxindependent mechanism in Arabidopsis. Plant Physiology, v.149, n.3, p.1579-1592, 2009.

DONOSO, E. Efecto de Trichoderma harzianum y compost sobre el crecimiento de plántulas de Pinus radiata em viveiro. Bosque, v.29, n.1, p.52-57, 2008.

DINIZ, K. A.; OLIVEIRA, J. A.; GUIMARÃES, R. M.; CARVALHO, M. L. M.; MACHADO, J. C. Incorporação de microrganismos, aminoácidos, micronutrientes e reguladores de crescimento em sementes de alface pela técnica de peliculização. Revista Brasileira de Sementes, v.28, n.3, p.37-43, 2006.

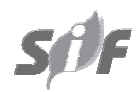

ETHUR, L. Z.; BLUME, E.; MUNIZ, M.; SILVA, A. C. F.; STEFANELO, D. R.; ROCHA, E. K. Fungos antagonistas a Sclerotinia sclerotiorum em pepineiro cultivado em estufa. Fitopatologia Brasileira, v.30, n.2, p.127-133, 2005.

CARVALHO FILHO, M. R.; MELLO, S. C. M.; SANTOS, R. P.; MENÊZES, J. E. Avaliação de isolados de Trichoderma na promoção de crescimento, produção de ácido indolacético in vitro e colonização endofítica de mudas de eucalipto. Brasília, DF: Embrapa Recursos Genéticos e Biotecnologia, 2008. (Boletim de Pesquisa e Desenvolvimento, 226)

FILHO, M. R. C.; MELLO, S. C. M. de; SANTOS, R. P. dos; MENÊZES, J. E. Avaliação de isolados de Trichoderma na promoção de crescimento, produção de ácido indolacético in vitro e colonização endofítica de mudas de eucalipto.

Boletim de pesquisa e desenvolvimento 226. Brasília, DF: Embrapa Recursos Genéticos e Biotecnologia, 2008.

FORTES, F. O.; SILVA, A. C. F.; ALMANÇA, M. A. K.; TEDESCO, S. B. Promoção de enraizamento de microestacas de um clone de Eucapyptus sp. por Trichoderma spp. Revista Árvore, v.31, n.2, p.221-228, 2007.

GLUFKE, C. Espécies florestais recomendadas para recuperação de áreas degradadas. Porto Alegre: Fundação Zoobotânica do Rio Grande do Sul, 1999. 48p.

HARMAN, G. E.; PETZOLDT, R.; COMIS, A.; CHEN, J. Interactions between Trichoderma harzianum Strain T22 and maize inbred line Mo17 and effects of these interactions on diseases caused by Pythium ultimum and Colletotrichum graminicola. Plant

Physiology, v.94, n.2, p. 146-153, 2004.

HARMAN, G. E. Myths and dogmas of biocontrol. Changes in perceptions derived from research on Trichoderma harzianum T-22. Plant Disease, v. 84, n.4, p.376-393, 2000.

HOYOS-CARVAJAL, L.; ORDUZ, S.; BISSETT, J. Growth stimulation in bean (Phaseolus vulgaris L.) by Trichoderma. Biological Control., v.51.p.409-416, 2009.

Revista Árvore, Viçosa-MG, v.39, n.1, p.167-176, 2015 
INBAR, M. J.; ABRAMSKY, D. C.; CHET, I. Plant growth enhancement and disease control bay Trichoderma harzianum in vegetable seedlings grown under commercial conditions. European Journal of Plant Pathology, v.100, n.5, p.337-346, 1994.

JYOTSNA, A. S.; SRIVASTAVA, A.; SINGH, R. P.; SRIVASTAVA, A. K.; SAXENA, A. K.; ARORA, D. $\mathrm{K}$. Growth promotion and charcoal rot management in chickpea by Trichoderma harzianum. Journal of Plant Protection Research, v.48, n.1, p.81-91, 2008.

KLEIFELD, O.; CHET, I. Trichoderma harzianum interaction with plants and effect on growth response. Plant and Soil. v.144, p.267-272, 1992.

LORENZI, H. Árvores brasileiras: manual de identificação e cultivo de plantas arbóreas nativas do Brasil. 2.ed. Nova Odessa: Plantarum, 1998. 89p.

MELO, I. S. Agentes microbianos de controle de fungos fitopatogênicos. In: MELO, I. S.; AZEVEDO, J. L., (Ed.). Controle biológico. Jaguariúna: Embrapa, 1998. v.1.p.17-60.

NAKAGAWA, J. Testes de vigor baseados na avaliação das plântulas. In: VIEIRA, R. D.; CARVAlHO, N. M. Testes de vigor em sementes. Jaboticabal: Funep, 1994. p.49-86.

OLIVEIRA, G. G. Trichoderma spp. no crescimento vegetal e no biocontrole de Sclerotinia sclerotiorum e de patógenos em sementes de cártamo (Carthamus tinctorius). 2007. 80f. Dissertação (Mestrado em Agronomia) - Universidade Federal de Santa Maria, Santa Maria, 2007.

OZBAY, N.; NEWMAN, S. E.; BROWN, W. M. The effect oh the Trichoderma harzianum strains on the growth of tomato seddlings. Acta Horticola. v.635, p.131-134, 2004.

OUSLEY, M. A.; LYNCH, J. M.; WHIPPS, J. M. Effect of Trichoderma on plant growth: a balance between inhibition and growth promotion. Microbial Ecology,. v.26, n.3, p.277-285, 1993.

PIÑA-RODRIGUES, F. C. M.; FREIRE, J. M.; SILVA, L. D. Parâmetros genéticos para colheita de sementes de espécies florestais. In: PIÑARODRIGUES, F. C. M. et al. (Org.). Parâmetros técnicos para produção de sementes florestais. Seropédica: Universidade Federal Rural do Rio de Janeiro, 2007. p.51-102.

PORTAL ACTION. Disponível em: http:// www.portalaction.com.br. Acesso em: 15 jun. 2011.

RESENDE, M. L.; OLIVEIRA, J. A.; GUIMARÃES, R. M.; PINHO, R. G. V.; VIEIRA, A. R. Inoculações de sementes de milho utilizando o Trichoderma harzianum como promotor de crescimento. Ciência e Agrotecnologia, v.28, n.4, p.793-798, 2004.

SILVA, L. D.; HIGA, A. R. Planejamento e implantação de pomares de sementes de espécies florestais nativas. In: HIGA, A. R.; SILVA, L. D. (Coord.). Pomar de sementes de espécies florestais nativas. Curitiba: FUPEF, 2006. p.13-39.

SILVA, F. A. S.; AZEVEDO, C. A. V. Principal components analysis in the software AssistatStatistical Attendance. In: WORLD CONGRESS ON COMPUTERS IN AGRICULTURE, 7., 2009, Reno. Proceedings...Reno-NV-USA: American Society of Agricultural and Biological Engineers, 2009.

TAvares, G. M. Podridão do pé do mamoeiro: infestação de solos de cultivo, controle alternativo com indutores de resistência e Trichoderma e avaliação dos mecanismos de defesa envolvidos. 2009. $121 \mathrm{f}$. Tese. (Doutorado em Fitopatologia) - Universidade Federal Rural de Pernambuco, Recife, 2009.

YEDIDIA, I.; SRIVASTVA, A. K.; KAPULNIK, Y.; CHET, I. Effect of Trichoderma harzianum on microelement concentrations and increased growth of cucumber plants. Plant and Soil, v.235, p.235-242, 2001. 